\title{
Field Analysis of Ridged Waveguides USING TRANSFER MATRIX
}

\author{
Wei-hua Zong ${ }^{1 a}$, Ming-xin Shao ${ }^{2 b}$ and Xiao-yun $\mathrm{Qu}^{3 \mathrm{c}}$ \\ ${ }^{1}$ Shandong Provincial Key Laboratory of Industrial Control Technology,School of Automation \\ Engineering, Qingdao University, Qingdao, China 266071 \\ ${ }^{2}$ Hisense Network Technology Co. Ltd., Qingdao, China 266071,
}

${ }^{3}$ China Aerospace Science and Technology Corporation No. 5 Academy No.513 , Institute, Yantai, China 264000,

Email: ${ }^{a}$ weihuazong@126.com, ${ }^{\text {m }}$ mxshao@126.com, ${ }^{c}$ selina.qu@163.com

Keywords- matrices; mode matching method; ridged waveguides; transfer matri

Abstract. The mode matching method is applied to analyze generaliz ridge ravegy des. The
tangential fields in each region are expressed in terms of the prod or eral m, ices, i.e., a
functional matrix about $\mathrm{x}-\mathrm{F}(\mathrm{x})$, a functional matrix about $\mathrm{y}-\mathrm{G}(\mathrm{y}) \mathrm{a}$ a columi to of amplitudes. The boundary conditions are transformed into a set of linear eo at by taking ne inner products of each element of $\mathrm{G}(\mathrm{y})$ with weight functions. Two types id idged veguide are calculated to validate the theory. Several new modes not reported in pr vious analysis presented.

\section{Introduction}

Ridged waveguides have many applications in microwa the well known fact that the cutoff frequency o. rectangular waveguide with the same outer dime sio s. Theoretical research on them has been continued steadily $[1,2,4-9]$. The analyais metho involve mode-matching technique $[1,2,5,6]$, quasi-static method [4], variation' $\mathrm{mg} d$ [7] a dintegral equations technique [8, 9]. All these approaches are only for the analy f ric sed waveguide with a certain structure, can not be used to analyze a group of ridged war ard tur ent shapes. In this paper, a general mode matching method is presented, apply to differ types of generalized ridged waveguides (shown in Figure 1). The waveguide is on ted by serting arbitrary number of rectangular ridges in the rectangular waveguic

To analyze the waveguide, the ucture is divided into $I$ regions. For each region $i\left(x_{i} \leq x \leq x_{i+1}\right.$, $y_{i} \leq y \leq y_{i}+b_{i}$ ), we vress tangential fields in each region in terms of the multiplication of several matrices, i.e., a $\mathrm{h}$ tonal $\mathrm{n}$ trix about $x-\mathbf{F}(x)$, a functional matrix about $y-\mathbf{G}(y)$ and a column vector of itudes the individual region. The boundary conditions of each pair of adjacent region are complis $d$ by taking the inner products of each element of $\mathbf{G}(y)$ with weight functio, ar linear equations are obtained. While neither the eigenvalues nor the amplitude. n be solved directly from the equations. The boundary conditions of the waveguide's wall are invo $\mathbb{A}$ to solve the problem. Two types of ridged waveguide are calculated to validate the theory. Sereral new modes not reported in previous analysis are presented.

\section{Matrix Formulation Of Field Components}

In general, the modes in ridged waveguide with inhomogenous dielectric filled are neither TE nor TM to the guide axis. In each region the fields can be expressed as a superposition of TE and TM modes of parallel planes. We denote $\phi_{h}^{(i)}$ and $\phi_{e}^{(i)}$ as $z$-components of the magnetic-type and electric-type Hertzian potentials of TE and TM modes in region $i$, respectively. They are assumed to be ( $e^{-j \beta z}$ is omitted in this paper). 


$$
\begin{aligned}
\phi_{h}^{(i)} & =\sum_{n=0}^{\infty}\left[A_{n}^{(i)} \sin k_{x n}^{(i)}\left(x-d_{i}\right)+B_{n}^{(i)} \cos k_{x n}^{(i)}\left(x-d_{i}\right)\right] \cdot \cos k_{y n}^{(i)}\left(y-y_{i}\right) \\
\phi_{e}^{(i)} & =\sum_{n=1}^{\infty}\left[C_{n}^{(i)} \sin k_{x n}^{(i)}\left(x-d_{i}\right)+D_{n}^{(i)} \cos k_{x n}^{(i)}\left(x-d_{i}\right)\right] \cdot \sin k_{y n}^{(i)}\left(y-y_{i}\right) \\
k_{y n}^{(i)} & =\frac{n \pi}{b_{i}}, \quad n=0,1,2 \ldots \\
k_{x n}^{(i)} & =\sqrt{\omega^{2} \varepsilon_{i} \mu_{0}-\left(k_{y n}^{(i)}\right)^{2}-\beta^{2}}, \quad n=0,1,2 \ldots
\end{aligned}
$$

where the upper symbol of $(i)$ represents region $i . A_{n}^{(i)}, B_{n}^{(i)}, C_{n}^{(i)}$, and $D_{n}^{(i)}$ are amplitude coefficients of each region. And $d_{1}=x_{1}, d_{I}=x_{I+1}, d_{i}=x_{i}$ (for $\left.1<i<I\right)$.

The amplitude coefficients are all unknown in previous

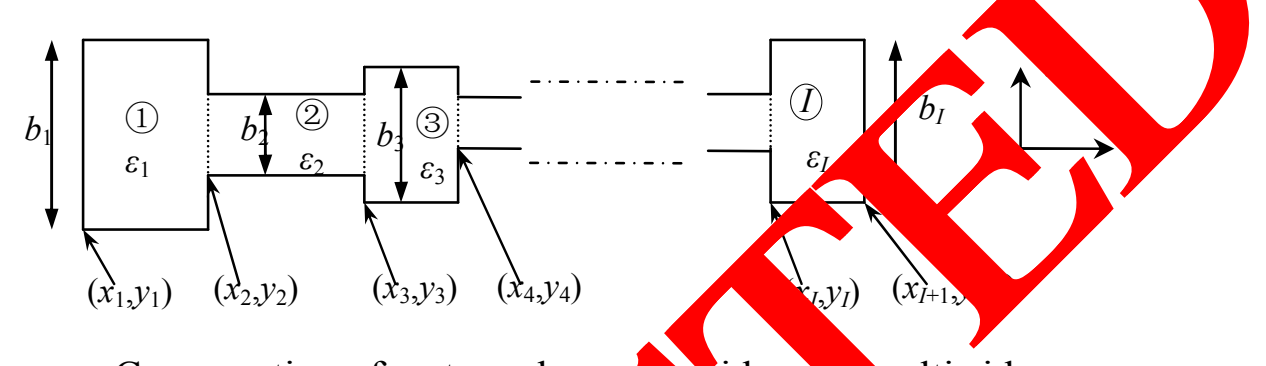

Figure 1. Cross section of rectangular yo raide wh vulti-ridge.

analysis. But in this paper, for regions 1 and $I$, some o the coefficients are of zero, determined by the boundary conditions at $x=x_{1}, x_{I+1}$ (which is perfe electric or magnetic walls). This will be discussed in section 4 .

Then the tangential field components in each aion are wnens as follows

$$
\begin{aligned}
E_{y}^{(i)}= & \sum_{n=0}^{\infty} k_{x n}^{(i)}\left[A_{n}^{(i)} \cos k_{x n}^{(i)}\left(x-d_{i}\right)-B_{n}^{(i)} \sin k_{x n}^{(i)}\left(x-d_{i}\right)\right] \cdot \cos k_{y n}^{(i)}\left(y-y_{i}\right)-\beta / \omega \varepsilon \\
& \left.+D_{n}^{(i)} \cos k_{x n}^{(i)}\left(x-d_{i}\right)\right] \cos k_{y n}^{(i)}\left(y-y_{i}\right) \\
E_{z}^{(i)} & =j\left(\beta^{2} / \omega \varepsilon_{i}-\omega \mu_{0}\right) \sum_{n=1}^{\infty}\left[C_{n}^{(i)} \sin k_{x n}^{(i)}\left(x-d_{i}\right) \sin k_{x n}^{(i)}\left(x-d_{i}\right)\right. \\
H_{y}^{(i)} & \left.=\left(\beta / \omega \mu_{0}\right) \sum_{n=1}^{\infty}\left[\left(A_{n}^{(i)} k_{y n}^{(i)}+D_{n}^{(i)} k_{x n}^{(i)}\right) \sin \alpha_{x n}^{(i)}\left(x-d_{i}\right)\right] \sin / y-y_{i}\right) \\
H_{z}^{(i)} & =j\left(\beta^{2} / \omega \mu_{0}-\omega \varepsilon_{i}\right) \sum_{n=0}^{\infty}\left[\left(A_{n}^{(i)} k_{x n}^{(i)}(\lambda) B_{n}^{(i)} \cos k_{x n}^{(i)}-d_{i}\right)\right] \cos k_{y n}^{(i)}\left(y-y_{i}\right)
\end{aligned}
$$

Because of th limita of com puting time and storage requirements, the infinite series terms in (4) are restrictea fip ce num er $N_{i}$. The expression in (4) can be written in matrix notation as

where

$$
\mathbf{A}^{(i)}=\left[\begin{array}{c}
A_{0}^{(i)} \\
\vdots \\
A_{N_{i}}^{(i)}
\end{array}\right], \mathbf{B}^{(i)}=\left[\begin{array}{c}
B_{0}^{(i)} \\
\vdots \\
B_{N_{i}}^{(i)}
\end{array}\right], \mathbf{C}^{(i)}=\left[\begin{array}{c}
C_{1}^{(i)} \\
\vdots \\
C_{N_{i}}^{(i)}
\end{array}\right], \quad \mathbf{D}^{(i)}=\left[\begin{array}{c}
D_{1}^{(i)} \\
\vdots \\
D_{N_{i}}^{(i)}
\end{array}\right]
$$

The components of $\mathbf{G}^{(i)}(y)$ and $\mathbf{F}^{(i)}(x)$ are as follows

$$
\mathbf{G}^{(i)}(y)=\left[\begin{array}{cccc}
\mathbf{G}_{1}^{(i)}(y) & \mathbf{0} & \mathbf{0} & \mathbf{0} \\
\mathbf{0} & \mathbf{G}_{2}^{(i)}(y) & \mathbf{0} & \mathbf{0} \\
\mathbf{0} & \mathbf{0} & \mathbf{G}_{3}^{(i)}(y) & \mathbf{0} \\
\mathbf{0} & \mathbf{0} & \mathbf{0} & \mathbf{G}_{4}^{(i)}(y)
\end{array}\right]_{4 \times\left(4 N_{i}+2\right)}
$$




$$
\mathbf{F}^{(i)}(x)=\left[\begin{array}{llll}
\mathbf{F}_{11}^{(i)}(x) & \mathbf{F}_{12}^{(i)}(x) & \mathbf{F}_{13}^{(i)}(x) & \mathbf{F}_{14}^{(i)}(x) \\
\mathbf{F}_{21}^{(i)}(x) & \mathbf{F}_{22}^{(i)}(x) & \mathbf{F}_{23}^{(i)}(x) & \mathbf{F}_{24}^{(i)}(x) \\
\mathbf{F}_{31}^{(i)}(x) & \mathbf{F}_{32}^{(i)}(x) & \mathbf{F}_{33}^{(i)}(x) & \mathbf{F}_{34}^{(i)}(x) \\
\mathbf{F}_{41}^{(i)}(x) & \mathbf{F}_{42}^{(i)}(x) & \mathbf{F}_{43}^{(i)}(x) & \mathbf{F}_{44}^{(i)}(x)
\end{array}\right]_{\left(4 N_{i}+2\right) \times\left(4 N_{i}+2\right)}
$$

where $\operatorname{diag}\left(f_{n}\right)_{n \in\left[n_{\min }, n_{\max }\right]}$ means a diagonal square matrix with element $f_{n}$ for $n=n_{\min }, n_{\min }+1, \cdots, n_{\max }$.

\section{Field Matching}

We define

$$
i_{+}=\left\{\begin{array}{ccc}
i & \text { if } & b_{i} \geq b_{i-1} \\
i-1 & \text { if } & b_{i}<b_{i-1}
\end{array}, i_{-}=\left\{\begin{array}{ccc}
i-1 & \text { if } & b_{i} \geq b_{i-1} \\
i & \text { if } & b_{i}<b_{i-1}
\end{array}\right.\right.
$$

then the boundary conditions at $x=x_{i}(i=2,3, \ldots, I)$ are given by

$$
\begin{aligned}
& E_{y}^{\left(i_{+}\right)}\left(x_{i}\right)=\left\{\begin{array}{cc}
0 & y \in\left[y_{i_{+}}, y_{i_{+}}+b_{i_{+}}\right] /\left[y_{i_{-}}, y_{i_{-}}+b_{i_{-}}\right] \\
E_{y}^{\left(i_{-}\right)}\left(x_{i}\right) & y \in\left[y_{i_{-}}, y_{i_{-}}+b_{i_{-}}\right]
\end{array}\right. \\
& E_{z}^{\left(i_{+}\right)}\left(x_{i}\right)=\left\{\begin{array}{cc}
0 & y \in\left[y_{i_{+}}, y_{i_{+}}+b_{i_{+}}\right] /\left[y_{i_{-}}, y_{i_{-}}+b_{i_{-}}\right] \\
E_{z}^{\left(i_{-}\right)}\left(x_{i}\right) & y \in\left[y_{i_{-}}, y_{i_{-}}+b_{i_{-}}\right]
\end{array}\right. \\
& H_{y}^{(i)}\left(x_{i}\right)=H_{y}^{(i-1)}\left(x_{i}\right), y \in\left[y_{i_{-}}, y_{i_{-}}+b_{i_{-}}\right] \\
& H_{z}^{(i)}\left(x_{i}\right)=H_{z}^{(i-1)}\left(x_{i}\right), \quad y \in\left[y_{i_{-}}, y_{i_{-}}+b_{i_{-}}\right]
\end{aligned}
$$

To transform the boundary conditions into an algeb aic system, we the the inner products of both sides of (9) with weight functions. Usually the ef nfunctions of the guide with larger height are chosen as the weight functions for enforcing the e tric contiy lity in (9.a) and (9.b), and the eigenfunctions of the smaller guide are chosen for magne dity in (9.c) and (9.d).

Taking the inner products of both sides o with $\mathbf{W}_{1}^{(i)}(y), \mathbf{W}_{2}^{(i)}(y), \mathbf{W}_{3}^{(i)}(y)$ and $\mathbf{W}_{4}^{(i)}(y)$, respectively, we can get matching equations. Co sid ing matrix form of fields in (5), the above process can be accomplished by takin the inner roducts of each element of $\mathbf{G}^{(i-1)}(y)$ and $\mathbf{G}^{(i)}(y)$ with same weight functions. The re ult $c_{\hat{a}}$ be writ yas

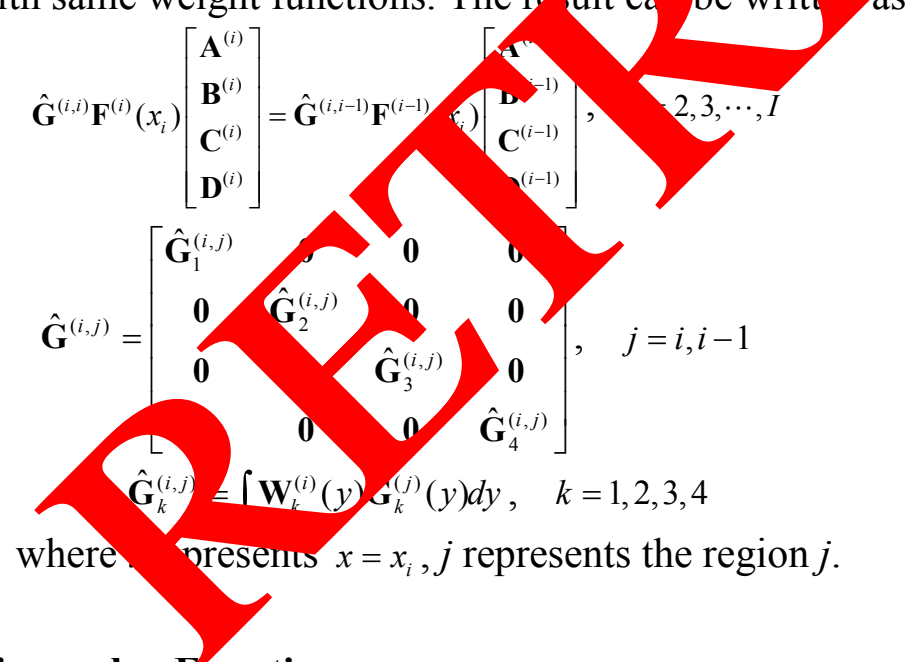

\section{Eigenvalue Equation}

The eigenvalues and unknown amplitudes cannot be solved directly from the linear equations of (10), because the number of equation is less than that of the amplitude. The boundary conditions at $x=x_{1}, x_{I+1}$ are used to determine the eigenvalues and amplitudes.

Let $N_{i} \equiv N \quad(i=1,2, \ldots, I)$, then $\hat{\mathbf{G}}^{(i, i)} \mathbf{F}^{(i)}\left(x_{i}\right)$ is a square matrix. By calculating the inverse of $\hat{\mathbf{G}}^{(i, i)} \mathbf{F}^{(i)}\left(x_{i}\right)$ in $(10)$, we get 
$\left[\begin{array}{l}\mathbf{A}^{(i)} \\ \mathbf{B}^{(i)} \\ \mathbf{C}^{(i)} \\ \mathbf{D}^{(i)}\end{array}\right]=\left[\mathbf{F}^{(i)}\left(x_{i}\right)\right]^{-1}\left[\hat{\mathbf{G}}^{(i, i)}\right]^{-1} \hat{\mathbf{G}}^{(i, i-1)} \mathbf{F}^{(i-1)}\left(x_{i}\right)\left[\begin{array}{l}\mathbf{A}^{(i-1)} \\ \mathbf{B}^{(i-1)} \\ \mathbf{C}^{(i-1)} \\ \mathbf{D}^{(i-1)}\end{array}\right]$

Let

$\mathbf{T}^{(i)}=\left[\mathbf{F}^{(i)}\left(x_{i}\right)\right]^{-1}\left[\hat{\mathbf{G}}^{(i, i)}\right]^{-1} \hat{\mathbf{G}}^{(i, i-1)} \mathbf{F}^{(i-1)}\left(x_{i}\right)$

$\mathbf{T}^{(i)}$ is the transfer matrix considering higher order modes for the region $i$. When cascading the transfer matrix of every pair of adjacent regions, we get

$\left[\begin{array}{l}\mathbf{A}^{(i)} \\ \mathbf{B}^{(i)} \\ \mathbf{C}^{(i)} \\ \mathbf{D}^{(i)}\end{array}\right]=\mathbf{T}^{(i)}\left[\begin{array}{l}\mathbf{A}^{(i-1)} \\ \mathbf{B}^{(i-1)} \\ \mathbf{C}^{(i-1)} \\ \mathbf{D}^{(i-1)}\end{array}\right]=\mathbf{T}^{(i)} \mathbf{T}^{(i-1)} \ldots \mathbf{T}^{(2)}\left[\begin{array}{l}\mathbf{A}^{(1)} \\ \mathbf{B}^{(1)} \\ \mathbf{C}^{(1)} \\ \mathbf{D}^{(1)}\end{array}\right]=\mathbf{M}^{(i)}\left[\begin{array}{l}\mathbf{A}^{(1)} \\ \mathbf{B}^{(1)} \\ \mathbf{C}^{(1)} \\ \mathbf{D}^{(1)}\end{array}\right]$

The amplitudes in the region $i$ are expressed in terms of the amplitudes perform calculation of eigenvalues, we express $\mathbf{M}^{(i)}$ with several submatrices

$$
\mathbf{M}^{(i)}=\left[\begin{array}{llll}
\mathbf{M}_{11}^{(i)} & \mathbf{M}_{12}^{(i)} & \mathbf{M}_{13}^{(i)} & \mathbf{M}_{14}^{(i)} \\
\mathbf{M}_{21}^{(i)} & \mathbf{M}_{22}^{(i)} & \mathbf{M}_{23}^{(i)} & \mathbf{M}_{24}^{(i)} \\
\mathbf{M}_{31}^{(i)} & \mathbf{M}_{32}^{(i)} & \mathbf{M}_{33}^{(i)} & \mathbf{M}_{34}^{(i)} \\
\mathbf{M}_{41}^{(i)} & \mathbf{M}_{42}^{(i)} & \mathbf{M}_{43}^{(i)} & \mathbf{M}_{44}^{(i)}
\end{array}\right]
$$

Considering boundary conditions at $x=x_{1}$ and $x=x_{I+1}$, we hav

$$
\left[\begin{array}{ll}
\mathbf{M}_{12}^{(I)} & \mathbf{M}_{13}^{(I)} \\
\mathbf{M}_{42}^{(I)} & \mathbf{M}_{43}^{(I)}
\end{array}\right]\left[\begin{array}{l}
\mathbf{B}^{(1)} \\
\mathbf{C}^{(1)}
\end{array}\right]=\mathbf{0}
$$

The above equations will have a nontrivial solution

$$
\left|\begin{array}{ll}
\mathbf{M}_{12}^{(I)} & \mathbf{M}_{13}^{(I)} \\
\mathbf{M}_{42}^{(I)} & \mathbf{M}_{43}^{(I)}
\end{array}\right|=0
$$

Equation (18) is the eigenvalue equation.

\section{Numerical Results}

Two examples are calculated to va,
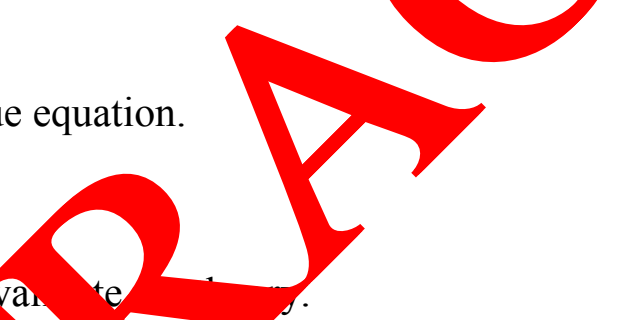

Ridged Waveguide with J momoge us Dielectric-Slab loading. The waveguide is shown in Figure 2. It has an aspect a of $b / a=0$. $1 \mathrm{~d}$ a dielectric slab of cross-sectional dimensions $a$ and $b$ is placed inside the ap bu en the 1idges, whereas the outer parts of the waveguide's cross section remain emr $y_{\text {. Figure } 3}$ w/s gap-height dependence of the cutoff wavelength $\lambda_{c}$ of the dominant mode ormal ed to the, waveguide broad dimension $a$ with $a=0.5 a$. The permittivity $\varepsilon_{r}$ severs as param Fig.3 that the data a well with those in [6].
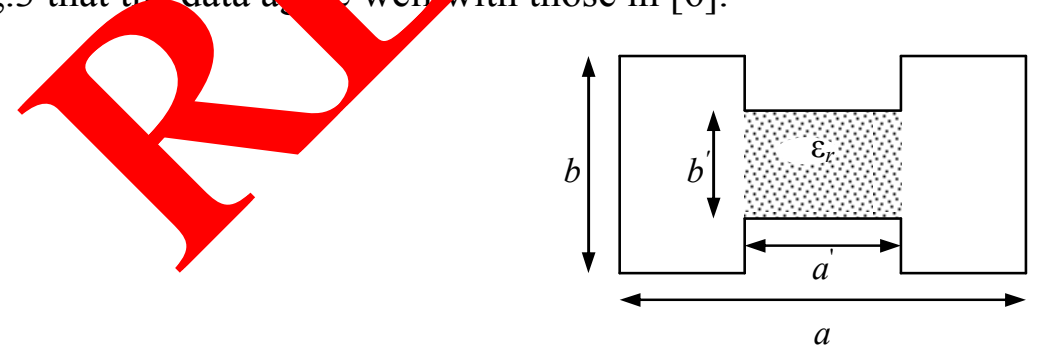

Figure 2. Cross section of ridged waveguide with inhomogeneous dielectric-slab loading 


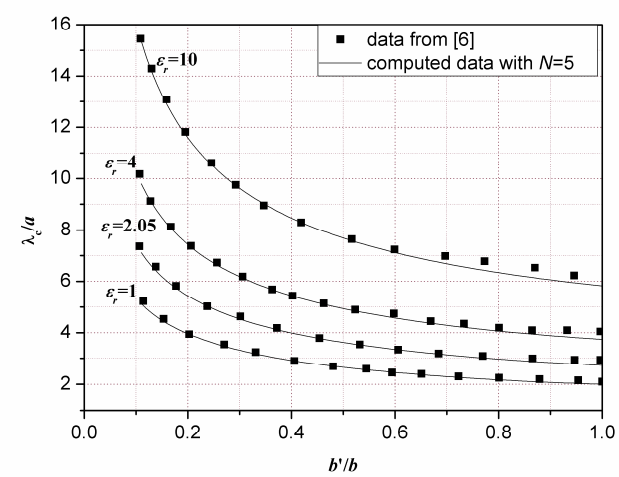

Figure 3. Gap-height dependence of cutoff wavelength $\lambda \mathrm{c}$ of the dominant mo norman $d$ to the waveguide broad dimension a

Single-Ridged Waveguide. The waveguide is filled with air, with dim cnsions of a $=9.0 \mathrm{~mm}$, $s=0.15 \mathrm{~mm}$, and $d=1.7 \mathrm{~mm}$ (see Figure 4). Using the symmetry of the y quid , we no cs can be classified into two types: 1 ) odd modes with a perfect magnetic wal $x=$ even odes with a perfect electric wall at $x=0$. We call the $k$ th odd mode as mode $k-1$, and $k$ th even mode as mode $2 k(k=1,2,3 \ldots)$.

Tables 1 and 2 show the cutoff wavenumbers of the firs of even and odd modes respectively, along with the data presented in [7, 8]. Th waveguide is aled in [7, 8] by use of variational method and integral equations technique espectively, whereas only odd modes are considered in [7].

As shown in able 1 ge agreement is good, except for mode 19. The calculated result of mode 19 is similar to th $\mathrm{TM} \mathrm{mo} 5$ in Table III of [8], whereas the datum is absent in [7]. In Table 2, the compr data 0 rode have much difference from [8]. There are corresponding data in [8] for node $16,20 \mathrm{a}, 22$, while no data for other modes are presented in [8]. It is found that the firsting of of 0.3332 in

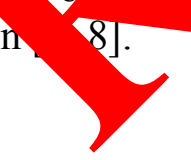


TABLE I. Cutoff WAVEnumbers (RAD/MM) Of The First ODD OdD Modes In A SINGLERIDGE WAVEGUIDES

\begin{tabular}{|c|c|c|c|}
\hline Mode m & $\begin{array}{c}\text { Present } \\
\text { Method } \\
(\mathrm{N}=10)\end{array}$ & Ref. [7] & Ref. [8] \\
\hline 1 & 0.0938 & 0.093 & 0.0926 \\
\hline 3 & 0.3332 & 0.3332 & 0.3332 \\
\hline 5 & 0.3817 & 0.3881 & 0.3811 \\
\hline 7 & 0.4710 & 0.4665 & 0.4711 \\
\hline 9 & 0.5277 & 0.5265 & 0.5263 \\
\hline 11 & 0.6654 & 0.6654 & 0.6653 \\
\hline 13 & 0.6916 & 0.6913 & 0.6916 \\
\hline 15 & 0.7407 & 0.7358 & 0.7410 \\
\hline 17 & 0.7457 & 0.7456 & 0.7453 \\
\hline 19 & 0.7480 & & 0.7481 \\
\hline 21 & 0.831417 & 0.8298 & 0.8295 \\
\hline
\end{tabular}

TABLE II. CUTOFF WAVENUMBERS (RAD/MM) OF THE FIRST F E SINGLE-RIDGE WAVEGUIDES

\section{Conclusions}

\begin{tabular}{|c|c|c|}
\hline Mode $\mathrm{m}$ & $\begin{array}{c}\text { Present Method } \\
(\mathrm{N}=10)\end{array}$ \\
\hline 2 & 0.3297 \\
\hline 4 & 0.3352 & \\
\hline 6 & 0.4691 \\
\hline 8 & 0.4713 \\
\hline 10 & & \\
\hline
\end{tabular}

The advantage of ne anproach is at the formulations can be used to analyze ridged waveguides with any shape nd the atching equations can be easily got from boundary conditions by simple matrix operation.

\section{Ackn ledg ents}

The won werma Zong was supported in part by Shandong Provincial Education Department, P. R. China Ver the International Cooperation Program for Excellent Lectures of 2009. 


\section{References}

[1] M. M. Fahmi, J. A. Ruiz-Crusz, R. R. Mansour, K. A. Zaki, "Compact ridge waveguide filter with arbitrarily placed transmission zeros using nonresonating nodes", IEEE Trans. Microwave Theory Tech, vol. 57, pp. 3354-3360, Dec., 2009.

[2] Ruiz-Cruz, J. A.; Zhang, Y.; Zaki, K. A.; Piloto, A. J.; Tallo, J., "Ultra-Wideband LTCC Ridge Waveguide Filters", IEEE Microwave and Wireless Components Letters, vol. 17, pp. 115-117, March, 2007.

[3] S. Bastioli, L. Marcaccioli, R. Sorrentino, "Waveguide Pseudoelliptic Filters Using Slant and Transverse Rectangular Ridge Resonators", IEEE Trans. Microw. Theory Tech., vol. 56, pp. 3129-3136, Dec., 2008.

[4] Y. Rong and K.A. Zaki, "Characteristics of generalized rectangular and circular ides", IEEE Trans. Microw. Theory Tech., vol. 48, pp. 258-264, March, 2000.

[5] G. Magerl, "Ridged waveguides with inhomogeneous dielectric slab loaam IEEE 1 ins. Microw. Theory Tech., vol. 26, pp. 413-416, June, 1978.

[6] Y. Utsumi, "Variational analysis of ridged waveguide modes", IEE $/$ Tran Mic , heory Tech., vol. 33, pp. 111-120, Feb. 1985.

[7] S. Amari, I. Bornemann and R. Vahldieck, "Application of a Cow d-Integ al-Equations Technique to ridged waveguide”, IEEE Trans. Microwave Th vech., v 44, pp. 2256-2264, Dec. 1996.

[8] W. Sun and C. A. Balanis, "MFIE analysis and desig ridge qveguides", IEEE Trans. Microw. Theory Tech., vol. 41, pp. 1965-1971, Nov. 993.

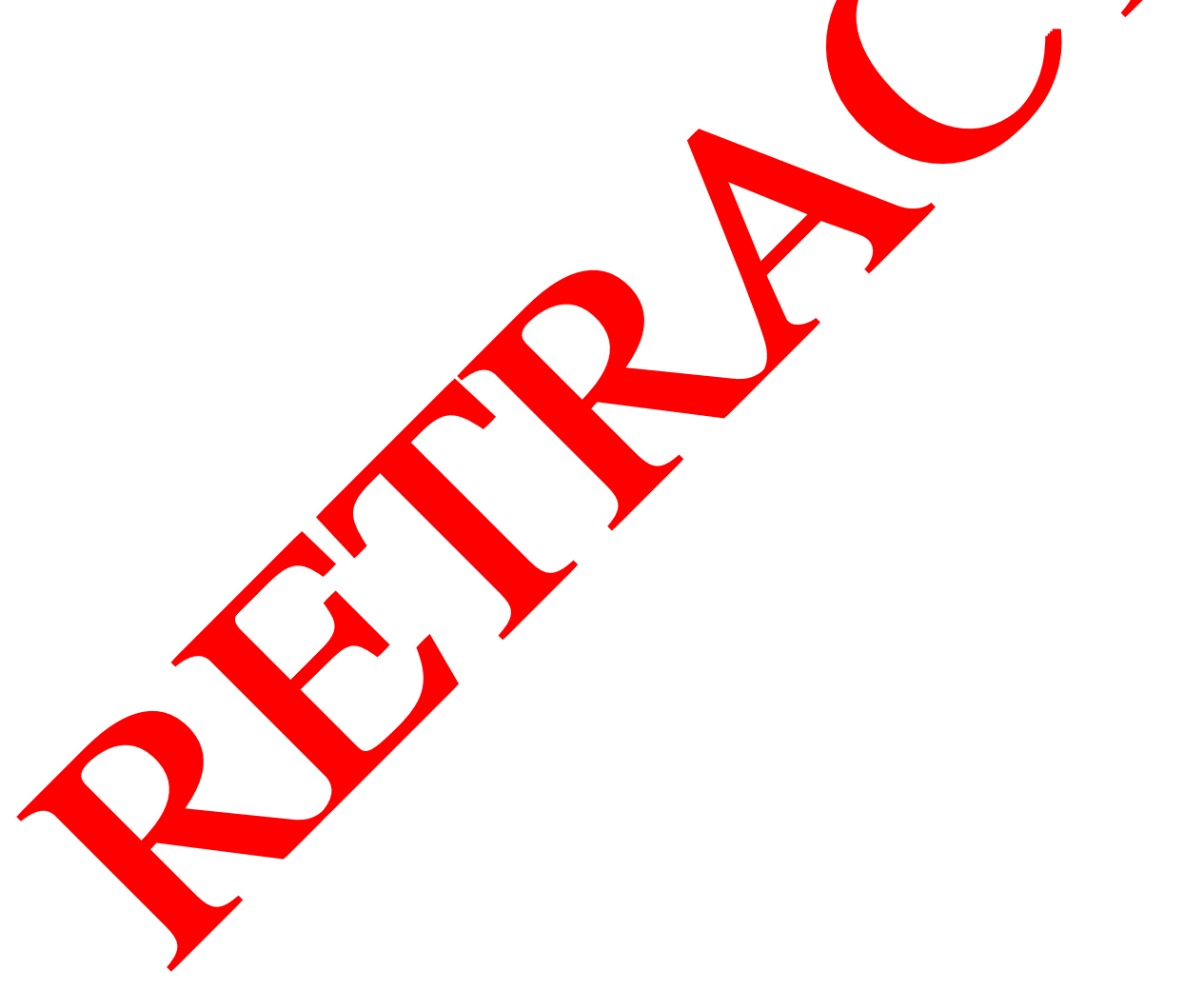

